\title{
A Flexible Approach to Motion Correction in Nuclear Medicine
}

\author{
K.Wells ${ }^{1}$, B. Goswami ${ }^{1}$, A.Abd Rahni ${ }^{1}$, J. Jones ${ }^{1}$ and M. Alowami ${ }^{1}$, E. Lewis ${ }^{1}$, M. Guy ${ }^{3}$ \\ ${ }^{1}$ Centre for Vision, Speech \& Signal Processing, Faculty of Engineering \& Physical Sciences, University of Surrey, Guildford, Surrey, UK \\ ${ }^{2}$ Department of Medical Physics, Royal Surrey County NHS Trust Hospital, Guildford, Surrey, UK
}

\section{INTRODUCTION}

There has been a considerable growth in the development of motion correction strategies for nuclear medicine over the last few years [eg 1, and references therein]. Methods have involved gating wherein only a single phase of the acquisition data is used, conformatory methods, requiring patients to breath in a trained manner, and more flexible approaches that have involved the tracking of external markers placed on the anterior chest surface or head. Other non-contact approaches have included using CT data to derive patient-specific models of the diaphragm that can be applied to correct PET emission data [2], or alternatively some authors propose methods which act on the emission data directly [3]. However, one of the major disadvantages of some approaches is that they may impose strong prior assumptions about expected respiratory motion. This may limit the accuracy of these approaches.

There has been anecdotal data published [1] as well data gathered within our own institution that suggests respiratory motion is a rather more complex process. We have observed subjects that tend to breath in a more shallow manner at the end of a study, compared to the, often, more erratic respiratory amplitude and phase at the beginning of a scan, where accurate motion correction is needed most. We have also found, albeit somewhat anecdotally, that subjects tend to breath in two modes [4]: some subjects exercise mainly the lower anterior surface near the stomach (abdominal breathers) whereas others may breath by elevating their upper chest (chest breathers). However, different/mixed modes of breathing can be observed at different time points during an individual emission scan. We have therefore sought to develop a more flexible approach to motion correction that can adaptively describe some of these aforementioned effects and flexibly correct for these processes in a variety of different circumstances. We propose to achieve this through a combination of patient-specific internal motion models, (derived from registration of dynamic CT data), and marker-less stereo tracking (to track external observed abdominal-chest motion), linked via a post-acquisition particle filtering framework. Particle filtering is an approach used for tracking applications- wherein a large number of 'particles' probabilistically represent the range of possible configurations of a system's state, in this case the internal organ configuration. This approach has found a wide range of non-medical applications from missile tracking to lip tracking. However, this is the first time this approach has been applied to nuclear medicine motion correction. This submission describes the overarching framework of our approach. Further details of the stereo tracking and abdominal chest surface modelling [5], organ-based registration work [6], and the particle filtering development [7] have also been submitted to this conference.

\section{PARTICLE Filtering PRINCIPLES}

Particle filtering provides a sub-optimal ${ }^{1}$ solution to predictively describe non-stationary non-linear processes. The

1 in the sense that the solution will be acceptable within the limits of the data, but may not represent a globally minimum cost solution. aforementioned facets of respiratory motion and motion correction fall into this class of problem. The key advantage to this postacquisition, data driven approach is that we do not make gross prior assumptions on the configuration of the hidden state of the system (i.e. the configuration of the internal organs at a certain time point during the emission scan). This uses a combination of marker-less tracking and a CT-based respiration model brought together in a particle filtering framework

First initiate our system by using a series of registrations between dynamic CT volumes of the subject demonstrating patientspecific respiratory motion. These define the initial patient-specific states of the system and the initial inter-state transitions, as well as a mapping between dynamic emission data and the hidden state (configuration).

During the SPECT/PET emission scan, we then infer an updated hidden state, or unobserved internal organ configuration, by using Monte Carlo sampling (and then filtering) of various estimated configuration propositions, or 'particles'. Such estimates are calculated using the previous state (of the internal organs) plus some noise or perturbation of the expected transition to the current state or configuration. We then compare an estimated anterior surface, (derived from the particles or propositions), with an observation of the actual surface (derived from a marker-less stereo camera system). By examining the differences between the particle or propositions' estimated surfaces and actual observed surface data, we can infer the current configuration of the internal organs. After an update step, the process is then repeated for all time points in the emission data. This allows the system to flexibly adopt previously unknown configurations of the internal organs, and thus allow for different modes of breathing (e.g. abdominal vs chestbased motion) to be represented.

The major advantage of such an approach is that the parameters used to describe the transitions to different states can be dynamically updated as the system evolves. Thus, we no longer rely on rigidly driving an assumed, possibly invariant, motion model through the emission data; but instead, we allow this to be inferred dynamically in-situ.

\section{Method DETAILS \& PRELIMINARY RESUltS}

Some further details and selected preliminary results are provided below on the main aspects of this approach.

In order to reduce the vast amounts of data produced at normal video rates by the marker-less tracking system, the camera system is polled at set intervals and a B-spline model of the chest is then fitted at set time points. The B-spline model regularises the number of points used to represent the anterior surface, making error estimates between observation and propagation model tractable. It also makes intra-state estimates possible and inter-subject comparison possible. Preliminary results using human volunteers can be seen in Figures 1 and 2. Further details of the marker-less tracking work and surface parameterization can be found in [5], submitted to this conference.

A patient specific propagation model that represents individual internal organ motion is derived from the use of dynamic low-dose CT data. Our previous work [8] has shown that low-dose CT data can be successfully segmented and used to construct attenuation maps of the abdominal-thoracic region. We are thus developing a 
similar approach to utilise dynamic CT data of internal respiratory motion, without increasing radiological dose above that received for a standard CT scan. Using a virtual dissection approach, major tissue groups seen in the CT data are segmented and labelled and then an Iterative Closest Point -based registration process is applied. Fig. 3 shows an exemplar plot comparing the quality of registration using ICP for the liver at different stages in the respiratory cycle, based on using the XCAT phantom, compared with no registration and the popular ITK-based non-rigid registration method. Further details appear in [6].

Preliminary work with the particle filtering (PF) framework [7] and the XCAT phantom has demonstrated that the PF approach can produce excellent tracking of internal point locations based on observation of external anterior markers over a wide range of respiratory periods and amplitudes. An exemplar plot appears in Fig. 4.

\section{CONCLUSIONS \& FURTHER WORK}

We have described the first occasion incorporating the particle filtering framework into motion correction for nuclear medicine. This relaxes the constraints often used in motion correction strategies, ad adaptively evolves to allow previously unkown states to be represented.

The final paper will include results from a simulation of the complete process undertaken using the XCAT phantom and provide early results of the application of the B-spline surface model and ICP-based registration work applied to clinical dynamic CT data alongside the development of a surface-based particle-filtering approach.

\section{References}

[1] D. Visvikis et al, (2006) NIM(A) Vol 569, pp 453-457

[2] S. McQuaid et al; (2008) NSS/MIC Conf Rec; pp 3651-3655

[3]W. Bai et al; Phys Med Biol 54 (2009) 2719-2736

[4] M. Alnowami (2008) MSc Dissertation; University of Surrey

[5] M. Alnowami et al (2009) submitted to MIC, Orlando, 2009

[6] J. Jones et al (2009) submitted to MIC, Orlando, 2009

[7] A. Ahb Rahni et al (2009) submitted to MIC, Orlando, 2009

[8] H. Kadhem et al, (2006) NSS/MIC Conf Vol:4, pp 2123-2127

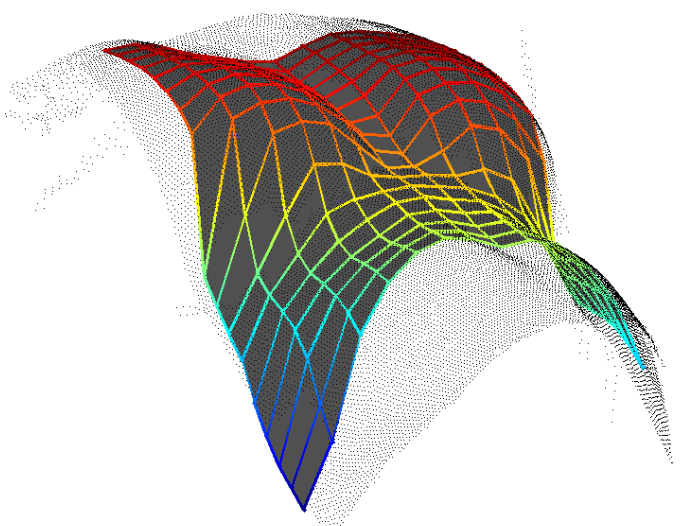

Figure 1 Grey high density points demonstrating data produced by the marker-less camera system used in this work. The mesh represents the control points used to define the uniformly-distributed B-spline representation of the surface shape.

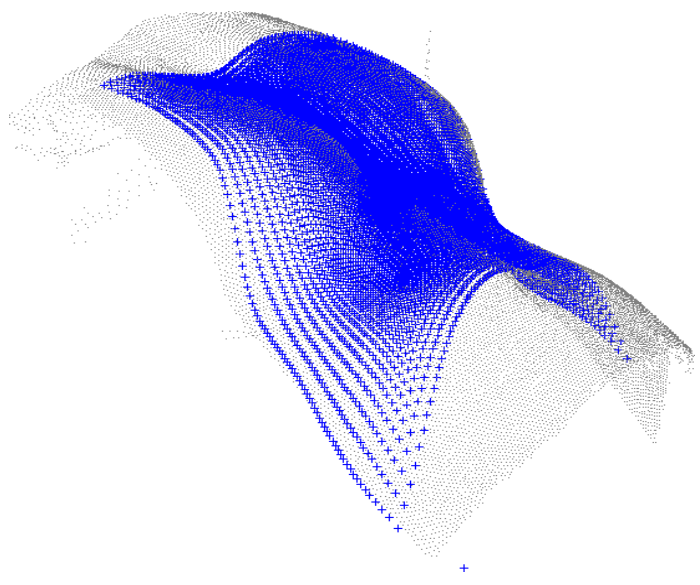

Figure 2 Camera surface data shown in grey with an interpolated surface of high density points produced from the B-spline representation shown in Fig. 1. This shows excellent visual agreement between model and camera data, although some significant matching errors (indiscernible in the figure) persist in the breast/pectoral region..

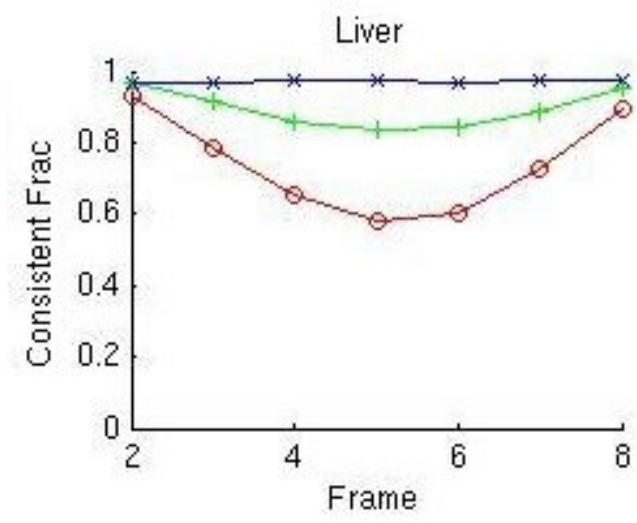

Figure 3. The Consistent Fraction (Intersection/Union of source and reference images) plotted as a function of frame number representing a respiratory cycle produced using the NCAT phantom. Blue: ICP registration, Green: ITK-based registration; Maroon: no registration. Further details appear in Jones, 2009.

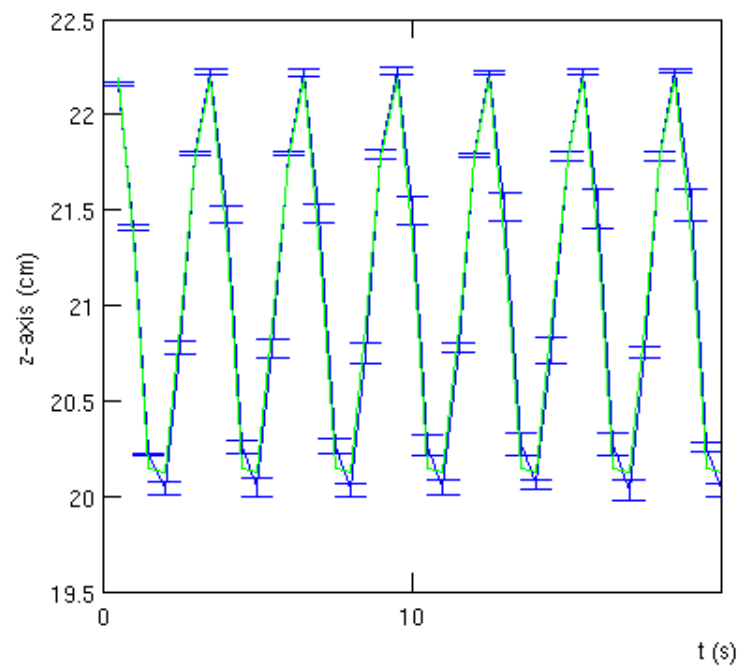

Figure 4. Exemplar plot of a simple application of particle filtering, using the NCAT phantom. In this case a single point on the top of the liver is tracked over a period of time during respiratory motion using a PF and using observations of a single point on the anterior chest surface. Green: true position of internal point on the liver. Blue: estimated position using PF. The error bars represent the standard deviations observed in the estimated position over a series of 10 trials of the PF. 